\title{
Diseño de cubiertas plegables experimentales bajo factores de cargas de viento y sismo
}

\author{
Design of experimental folding roofs under wind and earthquake load factors
}

Projeto de telhados experimentais dobráveis sob fatores de carga de vento e terremoto

Carlos Cesar Morales Guzmán

Arquitecto, Ph.D. en Arquitectura, Ph.D. en Estructuras.

Universidad Veracruzana, Veracruz, México.

dr.arqmorales@gmail.com

(iD) https://orcid.org/0000-0002-4499-6968

Recibido: agosto 28 de 2020

Aceptado: octubre 18 de 2020

Publicado: octubre 30 de 2020

\section{RESUMEN}

La siguiente investigación se desarrolló sobre las cubiertas plegables, donde se abordó el tema de los factores de cargas en dichas cubiertas, ya que este tipo de estructuras no se encuentra del todo normadas, por lo que se contemplan combinaciones experimentales de carga para el diseño de cubiertas no transitables, que se establecen en el Reglamento de Construcción del Distrito Federal (RCDF-2014), y el manual de diseño por vientos de CFE (MDVCFE-2015). Se revisa las cargas combinatorias con un software llamado WinTess, que realiza simulaciones estructurales contemplando una membrana estructural como rigidizador. El resultado será el dimensionamiento de la estructura.

Palabras clave: Combinaciones de Cargas, Simulación Estructural, Cubiertas Plegables

\section{ABSTRACT}

The following research was developed on folding roofs, where the issue of load factors in said roofs was addressed, since this type of structures is not fully regulated, so experimental load combinations are contemplated for the design of non-passable roofs, which are established in the Federal District Construction Regulations (RCDF-2014), and the CFE wind design manual (MDVCFE-2015). Combinatorial loads are reviewed with a software called WinTess, which performs structural simulations considering a structural membrane as a stiffener. The result will be the dimensioning of the structure.

Keywords: Load Combinations, Structural Simulation, Folding Covers

\section{RESUMO}

Foi desenvolvida a seguinte investigação em coberturas dobráveis, onde foi abordada a questão dos coeficientes de carga nas referidas coberturas, uma vez que este tipo de estrutura não se encontra totalmente regulamentado, pelo que são consideradas combinações experimentais de cargas para a concepção de Coberturas não transitáveis, que são estabelecidas no Regulamento de Construção do Distrito Federal 
(RCDF-2014) e no manual de projeto de vento CFE (MDVCFE-2015). As cargas combinatórias são revisadas com um software denominado WinTess, que realiza simulações estruturais considerando uma membrana estrutural como um reforçador. O resultado será o dimensionamento da estrutura.

Palavras-chave: Combinações de carga, Simulação estrutural, Tampas dobráveis

\section{INTRODUCCIÓN}

Se realizará la aplicación de las recomendaciones encontradas en los reglamentos para diseñar la estructura plegable, el cual se basa en el rango de factor de seguridad por resistencia y desplazamiento. El código de referencia será con base en el LRFD, que adopta un rango de seguridad para los miembros estructurales de 1 a 1.05 de resistencia del material (acero), por lo que se consideran los siguientes requerimientos de rendimiento para materiales isotrópicos: coeficiente de número de Poisson para el acero: .27 para diseñar, para más rigidez .30. Para determinar las cargas, se basará en las Normas Técnicas Complementarias sobre Criterios y Acciones para el Diseño Estructural de las Edificaciones del Reglamento de Construcciones para el Distrito Federal, las cuales especifican que la seguridad de una estructura deberá verificarse para el efecto combinado de acciones permanentes, variables y accidentales. Estas recomiendan que los efectos de todas las acciones deberán multiplicarse por un factor de carga igual a 1.1, aplicado a los efectos de todas las acciones que intervengan en la combinación, es decir, para revisar los efectos en estructuras en donde se incluyan movimientos sísmicos o presiones de vientos se deberán aplicar dichos factores. (Chilton 1998) (Chilton 2000) (Segui, 2000) (Morales, 2014a, 2016a, 2018a) (www. isc.cdmx.gob.mx)

Por lo que se selecciona las cargas recomendadas en dicha norma para cubiertas no transitables, que son las que se adapta más a nuestro tipo de estructura, dichos parámetros de carga son los siguientes: Carga Muerta: $30 \mathrm{~kg} / \mathrm{m} 2$, Carga Viva Máxima: $40 \mathrm{~kg} / \mathrm{m} 2$, Carga Viva Accidental: $20 \mathrm{~kg} /$ m2. En la Tabla 1 se desglosa la combinación de cargas que tendrá en la simulación estructural de la estructura, que afecta solo a las cubiertas no transitables (McCormac, 2000) (http://cgservicios. df.gob.mx/prontuario)
Tabla 1. Combinación de Cargas por el Reglamento de Construcción del D.F y el manual de LRFD. Fuente: (Morales, 2012, 2016a).

\begin{tabular}{ccccc}
\hline Combinación & $\mathbf{F}_{\mathrm{CM}}$ & $\mathbf{F}_{\mathrm{cV}}$ & $\mathbf{F}_{\mathrm{cS}}$ & $\mathbf{F}_{\mathrm{cV}}$ \\
\hline 1 & 1.1 & 1.1 & & \\
2 & 1.1 & 1.1 & $\operatorname{SISX}(1.1)+\operatorname{SISX}(.33)$ & \\
3 & 1.1 & 1.1 & $\operatorname{SISX}(1.1)-\operatorname{SISX}(.33)$ & \\
4 & 1.1 & 1.1 & $-\operatorname{SISX}(1.1)+\operatorname{SISX}(.33)$ & \\
5 & 1.1 & 1.1 & $-\operatorname{SISX}(1.1)-\operatorname{SISX}(.33)$ & \\
6 & 1.1 & 1.1 & $\operatorname{SISZ}(.33)+\operatorname{SISZ}(1.1)$ & \\
7 & 1.1 & 1.1 & $\operatorname{SISZ}(.33)-\operatorname{SISZ}(1.1)$ & \\
8 & 1.1 & 1.1 & $-\operatorname{SISZ}(.33)+\operatorname{SISZ}(1.1)$ & \\
9 & 1.1 & 1.1 & $-\operatorname{SISZ}(.33)-\operatorname{SISZ}(1.1)$ & \\
10 & 1.1 & 1.1 & & $\operatorname{VX}(1.1)$ \\
11 & 1.1 & 1.1 & & $-\operatorname{VX~(1.1)}$ \\
12 & 1.1 & 1.1 & & $\operatorname{VZ~(1.1)}$ \\
13 & 1.1 & 1.1 & & $-\operatorname{VZ~(1.1)}$ \\
\hline
\end{tabular}

Posteriormente, se verán todos los gráficos de tensión para verificar en dónde hay mayores esfuerzos y cómo estos interactúan dentro de la estructura, por lo que con esta información se desarrollarán las uniones adecuadas para resistir estos esfuerzos en sus uniones.

Para determinar cómo se resolverá la estabilidad de la estructura, se plantea una traslación geométrica sencilla. Esta geometría es fácil de realizar en este análisis, pues el sistema está constituido por una serie de marcos plegados en forma de " $X$ ". Esta forma ayudará a realizar mejor la simulación estructural. Para esta simulación, se especifica que el material será un acero A36, con un módulo de elasticidad de $2530 \mathrm{~kg} / \mathrm{cm}^{2}$ y un factor de Poisson de 0.3 con una densidad de $7.83847 \mathrm{Mton} / \mathrm{m}$. Las piezas seleccionadas son de tubos OCXE150 para miembros de unión principal; los atiesadores están conformados por la misma denominación, al igual que para las armaduras plegables en el espacio grande, donde se propusieron las mismas piezas tubulares, para no complicar tanto el análisis, ya que los miembros circulares tienen un radio de giro mayor y su excentricidad es óptima para el diseño de este tipo de estructuras. 
El resultado que veremos será una guía de cómo se comportan los esfuerzos en la estructura, en la que la simulación estática, y nos brindará una visión más clara de las dimensiones que pueden llegar a conformar estas cubiertas plegables y cuáles serán sus límites de claro a cubrir en un espacio determinado (Chudley 2001) (McCormac, 2000) (Morales, 2012, 2016a, 2018a)

Selección del Material. Para realizar el análisis estructural de la cubierta ligera retráctil, definiremos el material y el perfil con el que se trabajará. En esta etapa el material ideal es el aluminio estructural, ya que sus componentes de diferentes aleaciones hacen que sean resistentes y ligeros. La utilización de este material ha dado buenos resultados en otros proyectos de investigación, pero, para este caso, el factor económico nos afectó, y dado lo complicado que es mandar a generar una estructura de aluminio estructural en México, optamos en esta etapa de experimentación realizarla con acero A36 extraído del manual IMCA. De igual manera, las conexiones articuladas serán de un Acero A52, que son aún más resistentes. De ante mano sabemos los límites de resistencia que puede tener el acero; la desventaja es que su peso será un poco más que el aluminio, pero, para esta etapa, es crucial saber el comportamiento constructivo de la estructura.

Ya definido el material, configuremos la estructura. Dispondremos del manual IMCA. Este manual incluye las normas ASTM; con esto definimos las propiedades geométricas y de resistencia del dicho material. A continuación, se desglosa el tipo de miembros que se utilizaran:
Se realizarán con tubos OC (Circulares) ya que la propiedad geométrica de este tubo hace que sea más versátil su construcción y aporta mayor resistencia y estabilidad estructural.

Para la composición de nodos se harán armarán con tubos OC (Circulares), y placas de $1 / 4$ de espesor para la colocación de las piezas a articular.

La selección del perfil según su geometría y propiedades, se tomarán unos tubos $\mathrm{OC}$ que se especifican a continuación:

Tubo OC: unidades en $\mathrm{mm} \times \mathrm{mm}$. (Figura 1)

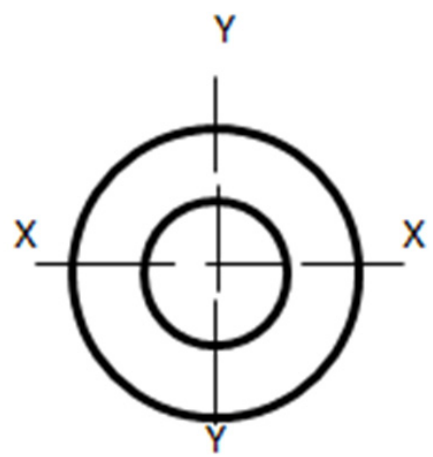

Figura 1. Determinación de la sección.

Fuente: Dibujo de elaboración propia. (Morales, 2012, 2016b)

El tubo circular OC, se selección de la Página de Tubo OC (IMCA), pág. 101-104, obteniendo los siguientes atributos geométricos y de resistencia del mismo miembro. (Tabla 2)

Tabla 2. Diámetros de tubos $O C$ propuestos para realizar simulaciones estructurales. Fuente: Elaboración propia y extraída del Manual IMCA. (IMCA, 2010) (Morales, 2012, 2016a, 2016b).

\begin{tabular}{|c|c|c|c|c|c|c|c|c|}
\hline \multirow{2}{*}{$\begin{array}{l}\text { Designación } \\
\text { Diámetro } D\end{array}$} & \multirow{2}{*}{$\begin{array}{l}\text { Diámetro } \\
\text { Nominal }\end{array}$} & \multirow{2}{*}{$\begin{array}{l}\text { Diámetro } \\
\text { Interior }\end{array}$} & \multirow{2}{*}{ PESO } & \multirow{2}{*}{ AREA } & \multicolumn{3}{|c|}{ Ejes $X-X$ y $Y-Y$} & \multirow{3}{*}{ Denominación } \\
\hline & & & & & I & $S$ & $r$ & \\
\hline $\mathrm{mm}^{*} \times \mathrm{mm}$ & in. & $\mathrm{mm}$ & $\mathrm{Kg} / \mathrm{m}$ & $\mathrm{cm} 2$ & $\mathrm{~cm} 4$ & $\mathrm{~cm} 3$ & $\mathrm{~cm}$ & \\
\hline $60 \times 8.74$ & 2 & 42.82 & 11.11 & 14.16 & 48.40 & 16.05 & 1.85 & 160 \\
\hline $73 \times 9.53$ & $21 / 2$ & 53.94 & 14.92 & 19 & 97.85 & 26.81 & 2.27 & 160 \\
\hline $89 \times 11.13$ & 3 & 66.94 & 21.35 & 27.19 & 209.80 & 47.20 & 2.78 & 160 \\
\hline $150 \times 5.78$ & $51 / 2$ & 143.45 & 30.27 & 39.45 & 862.75 & 125.90 & 4.45 & 160 \\
\hline
\end{tabular}

Notas: Se recomienda que al ordenar tubos de acero se especifique la norma oficial mexicana (NOM), el diámetro exterior y el espesor de pared; Se seleccionan cuatros diferentes perfiles de los cuales se realizará la simulación estructural y se colocará la más resistente a las combinaciones de cargas. 


\section{METODOLOGÍA}

La investigación destina a la estructura con un uso espacial multifuncional, ya que el modelo no cuenta con ninguna funcionalidad en específico. Estos parámetros se toman en cuenta porque el uso de los vientos dependerá de la localización del proyecto. En este caso, se utilizará la localización en Poza Rica, Veracruz, con una función espacial de nave industrial para determinar las cargas, con los siguientes parámetros:

Descripción. Para determinar las cargas horizontales que afectan a la estructura, se consideran vientos teorizados de los mapas de valores aproximados del Manual de Vientos de CFE. Primeramente, se recomienda en el manual que se cumpla con una seguridad necesaria de construcción, dependiendo de la función, para que se establezcan los niveles de importancia para la determinación de cargas; dichos niveles de velocidad se determinan para nuestro caso en el Grupo "B", ya que es una estructura para la que se destina una multifunción. El grado de seguridad es moderado. Este se clasifica en este grupo en el que aquellas, al fallar, generan baja pérdida de vidas humanas y que ocasionan daños materiales de magnitud intermedia, ya que el transito humano será de media influencia.

La clasificación de la estructura según su tipo de respuesta frente acciones del viento será de "Tipo -1 ". Esta categoría de estructuras es poco sensible a las ráfagas y a los efectos dinámicos del viento. Se agrupan en este tipo aquellas en las que la relación de esbeltez (definida como la relación entre la altura y la menor dimensión en planta) es menor o igual que cinco y con periodo natural de vibración del primer modo, menor o igual que un segundo. Se consideran dentro de este tipo la mayoría de los edificios para habitación u oficinas, bodegas, naves industriales, teatros y auditorios, puentes cortos.

Para determinar las presiones de diseño producidas por el viento sobre una cubierta plegable industrial con cubierta cilíndrica, se ubicará en la ciudad de Poza Rica, Veracruz, y se encuentra a 50 metros sobre el nivel del mar, con una temperatura media de $24.4^{\circ}$, aproximadamente. La nave se localiza en una zona urbana con obstrucciones de edificios entre 3 a $5 \mathrm{~m}$ de altura, (Figura 2) su geometría y dimensiones se muestran en la Figura. Los recubrimientos de la estructura son de membrana
Precontraint y estructura tubular OC. Su longitud se define por: $L=\pi(25) / 2=39.27 \mathrm{~m}$. (http:// cgservicios.df.gob.mx/prontuario) (Morales, 2013a, 2013b, 2016a, 2016b)

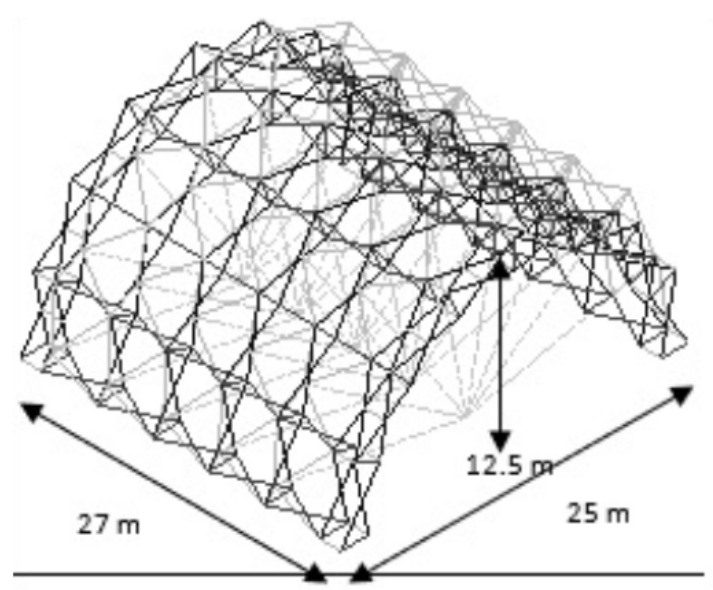

Figura 2. Descripción Geométrica de la Cubierta Fuente: Dibujo de Elaboración Propia (Morales 2012) (Morales 2013a, 2016b).

Procedimiento Inicial por Cargas por Viento. Por su importancia (inciso 4.1.3, del Manual de Procedimientos Constructivos de CFE), la estructura pertenece al Grupo B.

Determinación de la Velocidad de Diseño. La velocidad de diseño se determina con base en el Manual de Procedimientos Constructivos de CFE (inciso 4.2.). Dado que esta velocidad depende de varios parámetros, estos se calcularán como a continuación se indica.

Categoría de Terreno. De acuerdo con las características del terreno mencionado, se clasifica dentro de la Categoría 3 (Tabla 4.2.1, inciso 4.2.1. del Manual de Procedimientos Constructivos de CFE). Es de suponer que la rugosidad del terreno de los alrededores es uniforme, más allá de las longitudes mínimas establecidas en la misma Tabla.

Velocidad Regional. Considerando la región en donde se desplantará la nave, que pertenece al Grupo B, se obtiene del mapa de isotacas, para un periodo de retorno de 50 años:

$\mathrm{V}_{\mathrm{R}}=150 \mathrm{~km} / \mathrm{h}$, se optará la velocidad regional de Tuxpan ya que es la genera más afectaciones y solo se encuentra a una hora de Ciudad de Poza Rica. 
Factor de Exposición. El factor de exposición, $\mathrm{F}_{\mathrm{RZ}}$ es mayor de 10 metros; dado que la altura de punto foco es de 12.5 metros se realizará el cálculo de acuerdo con el inciso 4.2.3 del Manual de Procedimientos Constructivos de CFE. Por tanto, este factor vale:

Para encontrar: $F \alpha=F c \times F r z$, se encuentra en la Tabla 4.2.3 en el manual de CFE, por lo que Frz = $1.56[z / \delta] \alpha$ si $10<z>\delta$ por lo tanto Frz $=.91$

Factor de Topografía. Puesto que la nave industrial se desplantará en terreno prácticamente plano, el factor de topografía local (inciso 4.2.4. del Manual de Procedimientos Constructivos de CFE), le corresponde a: $F_{T}=0.9$

Velocidad de Diseño. La velocidad de diseño, que en este caso resulta constante en todo lo alto de la estructura, es (inciso 4.2_del Manual de Procedimientos Constructivos de CFE):

$\mathrm{V}_{\mathrm{D}}=0.9(0.86)(150)=\underline{116.1 \mathrm{~km} / \mathrm{h}}$

Presión Dinámica de Base. Dado que el sitio en donde se desplantará la estructura está prácticamente al nivel del mar, la presión barométrica que le corresponde es de $760 \mathrm{~mm}$ de Hg (Tabla 4.2.5. del Manual de Procedimientos Constructivos de CFE). Además, la temperatura anual media en este sitio es de $24.4^{\circ} \mathrm{C}$. Por tanto, el factor $G$ vale:

$G=0.392 / 273+\delta$

$\Omega=$ Presión barométrica en $\mathrm{mm}$ de Hg. (Tabla I.7. del Manual de Procedimientos Constructivos de CFE).

$\zeta=$ la temperatura anual en $C^{\circ}$ (se obtiene en usar las Tablas de medida de $\left.\mathrm{C}^{\circ}\right)$.

$G=.392(760) / 273+(24.4)=297.92 / 297.4=1$

Puesto que la altura de la estructura es menor de $10 \mathrm{~m}$, la presión dinámica de base es constante en toda su altura. Así, según el inciso 4.2.5 del Manual de Procedimientos Constructivos de CFE:

$q z=0.0048(1.0)(115.1)^{2}=64.7 \mathrm{~kg} / \mathrm{m}^{2}$. El factor 0.0048 corresponde a un medio de la densidad del aire.

Con esto, primera localización de cargas se determinan las presiones que tendrá la cubierta en su estructura, (Figura 3,4 ) el cual se integraran en el software WinTess que analizara cada combinación de carga dentro de la estructura, se obvia el desarrollo que sigue el manual por vientos de CFE, ya que el análisis de presiones de vientos que necesitamos para nuestro caso es solo la presión dinámica de base, el programa determina las presiones teóricas de afectaciones por viento dentro de la estructura, y para este caso solo realizaremos dicha simulación experimental. (http://cgservicios.df.gob.mx/prontuario) (Morales, 2013a, 2013b, 2016a, 2018a)
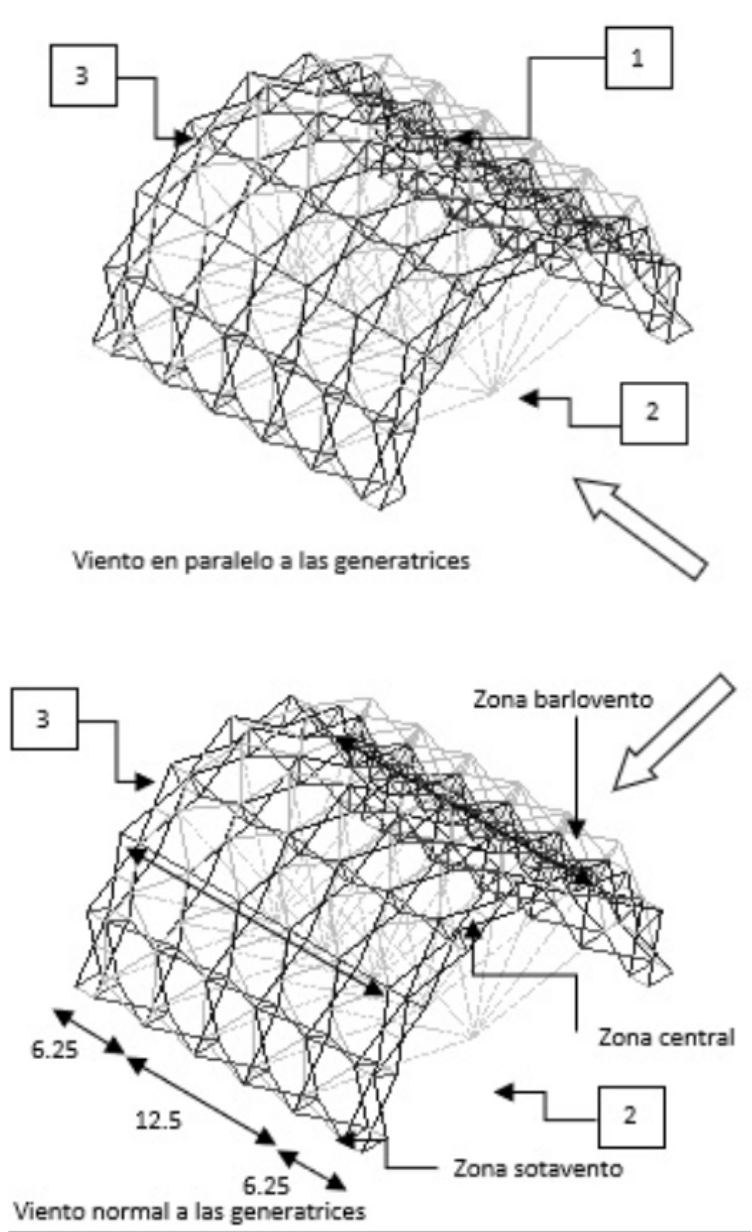

Figura 3, 4. Colocación de las Cargas de Viento en la zona de barlovento y sotavento, para presiones exterior 1: Cpe $=-.38$, para la zona presión interna 2: Cpe $=.8$ y para zona de presión interior de salida 3: Cpe $=.98$.

Fuente: Desarrollo como lo indica la norma del manual de vientos de CFE, (Morales, 2013a, 2016a, 2018a)

Procedimiento Inicial por Cargas por Sismo. En cuanto a las cargas horizontales por sismo, son estructuras que les afecta poco la carga sísmica, 
gracias a que sus conexiones nodales son altamente flexibles y puede mitigar este tipo de esfuerzos, sin embargo, para tener en orden la aplicación de las normas, se estableció aplicar la carga sísmica al proyecto bajo el RCDF.

Teóricamente, la edificación se encuentra localizada en Poza Rica, Veracruz, dentro de este estado hay dos tipos de zonas: la B y C; la estructura estará ubicada en la zona B. Ya definida la zona sísmica de ubicación, se aplicará el método de análisis estático sísmico simplificado, establecido en las normas complementarias del RCDF, en los que se deben cumplir los requisitos establecidos en la sección 2.2. Para calcular las fuerzas cortantes a diferentes niveles de una estructura, se supondrá un conjunto de fuerzas horizontales actuando sobre cada uno de los puntos en el supuesto concentradas las masas.

Cada una de estas fuerzas, será equivalente al peso de la masa que corresponde, multiplicado por un coeficiente proporcional a $h$, siendo $h$ la altura de la masa en cuestión sobre el desplante (o nivel a partir del cual las deformaciones estructurales pueden ser apreciables). El coeficiente aplicará de tal manera, que la relación $V_{o} / W_{o}$ sea igual a $c / Q^{\prime}$ pero no menor que $a_{o^{\prime}}$ donde $a_{0}$ es la ordenada espectral que corresponde a $T=0$ y $c$ el coeficiente sísmico. $a_{0}$ y $c$ se señalan en la Tabla 3.1. De las normas complementaras RCDF.

De acuerdo con este requisito, la fuerza lateral que actúa en el $i$-ésimo nivel, $F_{i}$, resulta ser:

$$
F_{i}=\frac{c}{Q}, W_{i} h_{i} \frac{\sum W_{i}}{\sum W_{i} h_{i}} ; \quad \frac{c}{Q}, \geq a_{o}
$$

\section{Dónde:}

Wi peso de la i-ésima masa; y

hi altura de la i-ésima masa sobre el desplante.

Para cumplir con la anterior fórmula, se buscará el comportamiento sísmico $Q$ de la norma; se adoptarán los valores especificados en alguna de las secciones siguientes, según se cumplan los requisitos en ellas indicados.

Se usará $Q=1.5$ ya que la estructura es resistente a las fuerzas laterales, gracias a las articulaciones que disipan la carga horizontal de efectos sísmicos, también se consideró el efecto del material también resistente a las torsiones y giros por los efectos de cargas horizontales.
Teniendo los parámetros anteriores, se procede a realizar el cálculo sísmico en la estructura, detectando sus cortantes críticos, que tendrán interacción con la estructura; por consiguiente, se analiza la estructura (esta solo tendrá un solo nivel de piso) mediante la factorización necesaria.

$\mathrm{c} / \mathrm{Q}>\mathrm{a}_{\mathrm{o}}=.21 / 2>0.06$ por lo tanto $.105>.06$, posteriormente, se junta con la formula sísmica estática.

$\mathrm{Fi}=\mathrm{c} / \mathrm{Q} \times \mathrm{Wi}=.105 \times(36.97$ Peso Propio de la Cubierta) $=3.8$ Ton, la cortante sísmica se distribuirá en la geometría de la estructura en las direcciones $\mathrm{X}$ y $\mathrm{Y}$

Este análisis de carga solamente será una guía para verificar si los desplazamientos y deformaciones en el programa y no sobrepasen a lo establecido por la norma que en nuestro caso será un desplazamiento de $50 \mathrm{~mm}$ para estructuras de acero. Posteriormente, ya definidas las premisas y parámetros anteriormente mencionados, se consideran qué datos se colocarán en la simulación, para que los resultados sean lo más cercanos posible a la realidad. Es por ello por lo que se tomaron muy en cuenta las recomendaciones de los reglamentos, ya que, los sistemas plegables dentro del país, es un campo novedoso para explorar, y relativamente nuevo para norma. (www. isc.cdmx.gob.mx) (Morales, 2013a, 2016a, 2018a)

\section{Características delas Estructuras Transformables.}

Para realizar un cálculo de esfuerzo en estos sistemas transformables toca el turno de razonar con el análisis de las siguientes ecuaciones (1) y (2) las expresiones de los desplazamientos en coordenadas locales Ecuación 1 (Figura 5). En función de estos desplazamientos, se calcularán para resolver el sistema de ecuaciones $L=S$ X X y así podremos calcularlos a partir de (1) los esfuerzos en las barras.

$$
\begin{array}{ll}
N_{1}=\frac{E \cdot A}{L_{1}} u_{1} & P_{1}=\frac{3 E I_{1} L}{L_{1}{ }^{2} L_{2}{ }^{2}} \quad v \\
N_{2}=\frac{E \cdot A}{L_{2}} u_{2} & P_{2}=\frac{3 \cdot E \cdot I_{2} \cdot L}{L_{1}{ }^{2} \cdot L_{2}{ }^{2}} \cdot w
\end{array}
$$

Figura 5. Ecuación 1. Deformación y Desplazamiento Fuente: Extraído de la revista Informes de la Construcción, (Escrig, F. 1988) 
Generalmente, es de mayor interés calcular los esfuerzos cortantes y los momentos flectores, Ecuación 2 (Figura 6) que las propias fuerzas trasversales, por ello se muestran las ecuaciones y condiciones que pueden llegar a tener las barras para estabilizar su forma (Ecuación 3, Figura 7). (Escrig, F. 1988) (Morales, 2013b, 2019)

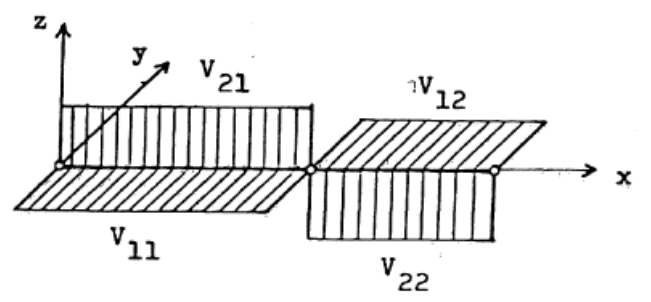

$$
\begin{array}{ll}
\mathrm{V}_{11}=\frac{\mathrm{P}_{1} \mathrm{~L}_{2}}{\mathrm{~L}} & \mathrm{~V}_{12}=\frac{\mathrm{P}_{1} \mathrm{~L}_{2}}{\mathrm{~L}} \\
\mathrm{~V}_{21}=\frac{\mathrm{P}_{2} \mathrm{~L}_{2}}{\mathrm{~L}} & \mathrm{~V}_{22}=\frac{\mathrm{P}_{2} \mathrm{~L}_{1}}{\mathrm{~L}}
\end{array}
$$

Figura 6. Ecuación 2, Esfuerzos Cortantes.

Fuente: Extraído de la revista informes de la construcción (Escrig, F. 1988)

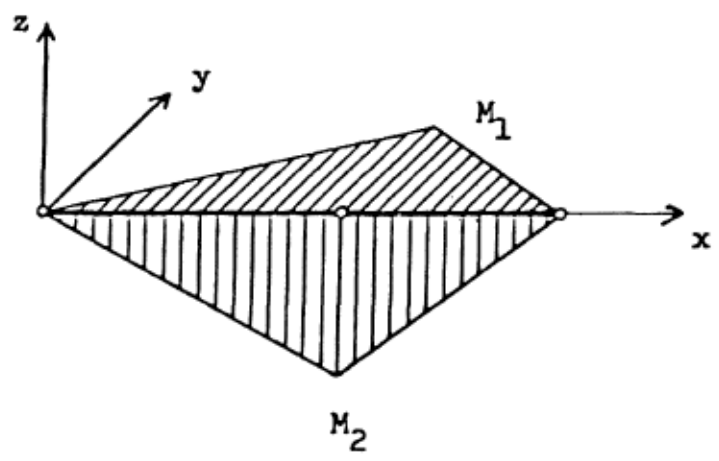

$$
M_{1}=\frac{P_{1} L_{1} L_{2}}{L} \quad M_{2}=\frac{P_{2} L_{1} L_{2}}{L}
$$

Figura 7. Ecuación 3, Esfuerzos de Momentos Fuente: Extraído de la revista informes de la construcción (Escrig, F. 1988)

\section{PARÁMETROS Y RESULTADOS DE LA SIMULACIÓN ESTRUCTURAL}

En esa fase la cubierta se le integra la membrana. Hay varios métodos para hacerlo. En este caso será por el método virtual, donde se obtendrá la forma. Para ello, se apoya con el uso de un software institucional elaborado por el Dr. Ramón Sastre Sastre, profesor de la Escuela Técnica Superior de Arquitectura en la Universidad Politécnica de Catalunya.

Dicho software se llama WinTess versión 3.11. Realiza el método virtual por densidades de fuerza. Este método genera una relajación en la membrana para obtener la forma deseada de la cubierta textil (Figuras 8,9 ), en la que primeramente se genera una malla rectangular de $35 \mathrm{~m}$ de ancho, que sería la longitud que tendría si fuese una cubierta rígida curva, y $27 \mathrm{~m}$ de largo que tiene la cubierta en su dirección lineal. (Morales, 2013a, 2018b, 2019b)

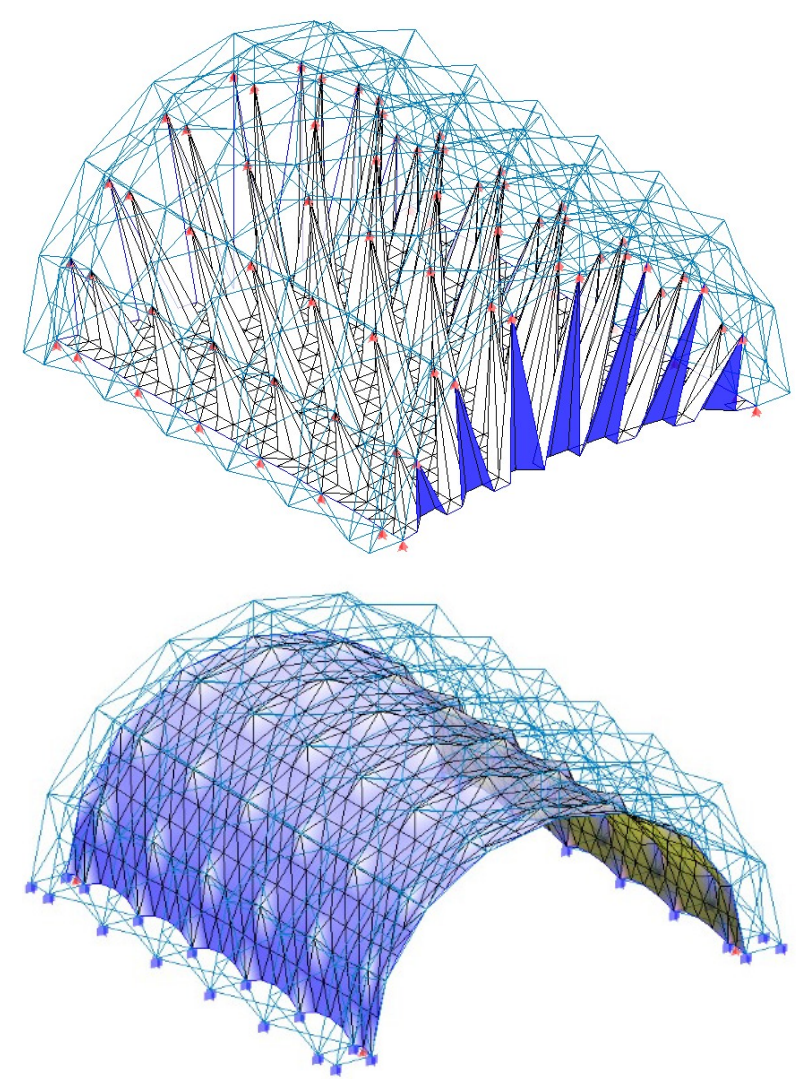

Figuras 8, 9. La simulación por software nos ayuda a realizar más rápido la obtención de la forma.

Fuente: Extraído de Estancia Post Doctoral (Morales, 2013a).

Previamente realizado el análisis de combinaciones de carga, se selecciona la combinación de cargas que más afecta a la estructura y se estudia el caso. WinTess puede realizar casos de combinaciones de carga por separado o en conjunto, que posteriormente se pueden combinar los resultados 
y ver qué tipo de cargas son las que más afectan al sistema estructural, en consecuencia, quedaría así la carga que más afecto a la estructura (Tabla. 3):

Tabla 3. Combinación más afectada que se calculó en el programa WinTess. Fuente: Morales, 2016a, 2019).

\begin{tabular}{l}
\hline WINTESS \\
\hline Combinación de Carga: CM $1.1+$ CV $1.1+1.1$ VIEN X \\
Sobrecarga $=20 \mathrm{~kg} / \mathrm{m}^{2}+$ Carga Accidental: $40 \mathrm{~kg} / \mathrm{m}^{2}$ \\
Viento $X=116.1 \mathrm{~km} / \mathrm{hViento} \mathrm{Y}=64.7 \mathrm{~km} / \mathrm{h}$ Viento total $=132.91 \mathrm{~km} / \mathrm{h}$ \\
Tipo de edificio $=$ Abierto $(3)>\mathrm{Sin} \mathrm{c}=0$ \\
Pretensado de la membrana $=0.08 / 0.08 \%$ \\
Peso de los cables $=791.8 \mathrm{~kg}$ \\
Peso de los tubos $=35376.0 \mathrm{~kg}$ \\
Peso de la membrana $=803.7 \mathrm{~kg}$
\end{tabular}

Para este caso, se seleccionó una membrana Serge Ferrari-Fluitop-T2-1002, con una resistencia de $\mathrm{RK}(\mathrm{daN} / 5 \mathrm{~cm}) 420 / 420$, y los tubos circulares de $150 \mathrm{~mm}$ de diámetro y $5 \mathrm{~mm}$ de espesor de acero con denominación europea S235. Solo se colocará el resultado más óptimo de la simulación de los miembros estructurales anteriormente mencionado. Se desprecian las otras medidas, ya que no resisten el mínimo de factorización de seguridad. Los cables de acero galvanizado se especifican de $18 \mathrm{~mm}$ de espesor. Ya realizado el cálculo sobre estas especificaciones, se verifican los datos arrojados del programa con las normas anteriormente mencionadas, que están incluidas en el programa de cálculo.

En la Tabla. 4 se observa que todas las barras con mayor tensión no superan el Ratio de seguridad establecido a la unidad (1), se menciona que, para el tipo de estructura, la comprobación sigue siendo la unidad, pero, en algunos casos, la comprobación de seguridad puede dejarse a 1.65 en los tubos, ya que este tipo de estructura no es transitable y esto genera mucha controversia.
Especialistas como el Dr. Ramón Sastre Sastre y el Dr. José Ignacio Llorens Duran, están redactando reglamentaciones para este tipo de estructuras en Europa y han comprobado que dichas estructuras son factibles con un factor de seguridad de 1,65. Es posible para considerar la aleatoriedad en las cargas y en las incertidumbres del modelo de análisis en relación con la geometría real. Sin embargo, en este ejercicio, el Ratio se dejó a la unidad por que se manejan factores parciales para cargas y resistencias por la falta de información de este tipo de estructuras transformables. $Y$, en nuestro caso, la geometría debe tener rigidez a la hora de estar completamente desplegada y mantenerse en la misma posición para estar en el rango de la seguridad. (Morales, 2016a, 2016b, 2017, 2018a, 2019)

Los momentos en la cubierta siguiendo bajos (Figura 10), ya que la tensión que domina son los axialmente en la estructura, pues su configuración estructural actúa como vigas en celosía. Esto hace que solo actúen los miembros diagonalmente en su máxima capacidad, y que funcione mucho mejor el sistema estructural, y aunque se note que los axiles aumentaron por la misma presión del viento, que choca con la membrana, con ello aumenta podría aumentar la dimensión de la barra si se trasladara la cubierta a otro sitio. En cuanto al desplazamiento por deformación, no superó los $50 \mathrm{~mm}$; el rango alcanzado fue de $45 \mathrm{~mm}$ de desplazamiento por deformación óptimo para este tipo de estructuras de acero.

Tabla 4. Cuadro de esfuerzos y Ratios de seguridad. Fuente: (Morales, 2016b, 2019).

\begin{tabular}{|c|c|c|c|c|c|c|c|}
\hline \multicolumn{8}{|c|}{ DATOS BARRAS RÍGIDAS } \\
\hline Barra & Axial & Torsor & M.max & C.max & Tensión & Ratio & \\
\hline & $\mathbf{T}$ & Tm & Tm & $\mathbf{T}$ & $\mathrm{kg} / \mathrm{cm}^{2}$ & & \\
\hline 1993 & -20.120 & 0.026 & 0.233 & 0.014 & -1047.9 & 1.16 & $\varnothing 150 \cdot 5 \_S 235$ \\
\hline 2066 & 15.497 & 0.015 & 0.214 & 0.026 & 1349.6 & 0.95 & $\varnothing 150 \cdot 5 \_S 235$ \\
\hline 2074 & -16.809 & 0.026 & 0.211 & 0.004 & -845.1 & 0.99 & Ø150.5_S235 \\
\hline 2454 & -17.473 & 0.000 & 0.181 & 0.074 & -933.2 & 0.98 & $\varnothing 150 \cdot 5 \_S 235$ \\
\hline 2462 & 19.036 & 0.002 & 0.252 & 0.123 & 1371.3 & 0.96 & $\varnothing 150 \cdot 5 \_S 235$ \\
\hline 2467 & 16.540 & 0.003 & 0.189 & 0.077 & 1373.9 & 0.96 & $\varnothing 150 \cdot 5 \_S 235$ \\
\hline 2474 & -19.051 & 0.007 & 0.250 & 0.122 & -779.4 & 0.99 & $\varnothing 150 \cdot 5 \_S 235$ \\
\hline 2502 & 16.507 & 0.004 & 0.194 & 0.079 & 1373.5 & 0.96 & Ø150.5_S235 \\
\hline 2505 & -17.294 & 0.000 & 0.175 & 0.072 & -925.2 & 0.97 & Ø150.5_S235 \\
\hline 2509 & -18.468 & 0.008 & 0.265 & 0.129 & -738.6 & 0.97 & $\varnothing 150 \cdot 5 \_s 235$ \\
\hline
\end{tabular}




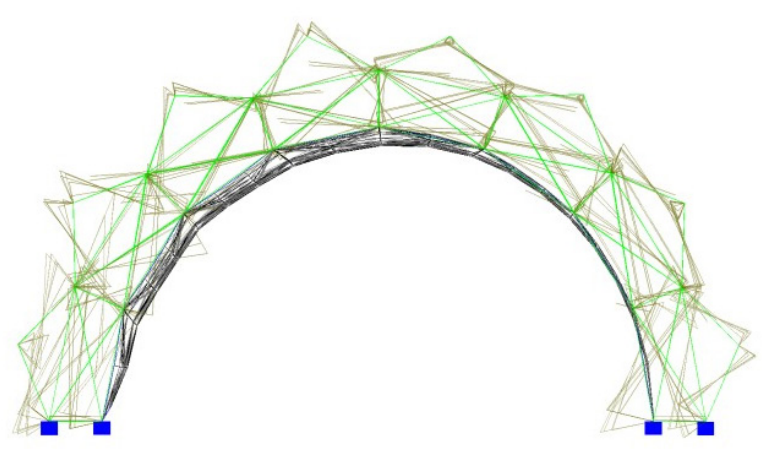

Figuras 10. Los momentos en la cubierta son muy bajos, ya que la Figura de la tijereta funciona como celosía. Fuente: Extraído de la Revista de la Arquitectura, Universidad Católica de Colombia (Morales, 2016.b)

Siguiendo con los resultados de los análisis del programa, podemos verificar también la seguridad estructural del cable y de la membrana textil. Este tipo de miembros trabajan solo a tracción; se debe tener cuidado para saber cuánta tensión pueden soportar, por esto la importancia de conocer las propiedades de los materiales y su módulo de elasticidad de los materiales a utilizar, pero para nuestro caso no hubo ningún problema de fallo estructural (Tabla 5).

Tabla 5. Dimensiones que se deben contemplar para el diseño constructivo de las conexiones de la estructura. Fuente: (Morales, 2016b)

\begin{tabular}{ccccc}
\hline \multicolumn{5}{c}{ TRACCIÓN MÁXIMA EN LA MEMBRANA } \\
\hline Barra & Nudos & T/metro & $\mathbf{k g} / 5 \mathbf{c m}$ & Ratio \\
\hline 1728 & $487-519$ & 1.470 & 73.5 & 0.90 \\
\hline \multicolumn{4}{c}{ TRACCIÓN EN LOS CABLES DE RELINGA } \\
\hline Cable & $\mathbf{T}$ & Ratio & Barra \\
\hline 1 & 2.860 & 0.75 & $\begin{array}{c}1,2,3,4,5,6,7,8,9,10,11,12,13,14,1 \\
\text { WS-2-(18mm)Galv }\end{array}$ \\
2 & 2.857 & 0.89 & $\begin{array}{c}45,46,47,48,49,50,51,52,53,54,55,5 \\
\text { WS-2-(18mm)Galv }\end{array}$ \\
3 & 1.593 & 0.65 & $\begin{array}{c}65,66,67,68,69,70,71,72,73,74,75,7 \\
1 \times 91-(18 m m) I n o x\end{array}$ \\
\hline
\end{tabular}

En cuanto a la membrana Serge Ferrari-FluitopT2-1002, resistió perfectamente las deformaciones por las presiones de viento, por empuje y succión que se colocaron dentro del programa, Para tener una mayor confiabilidad de los resultados, se puede observar en la Figura 11 los Ratio de los tubos, cables y membranas interactuando entres sí, y que ninguno se excedió del factor de seguridad (color rojo), pero en ese caso, la mayor solicitud la tuvieron las de color naranja, así que la estructura está en el rango de seguridad. (Berger 1996) (Morales, 2013a, 2016b, 2018a, 2018b, 2019)

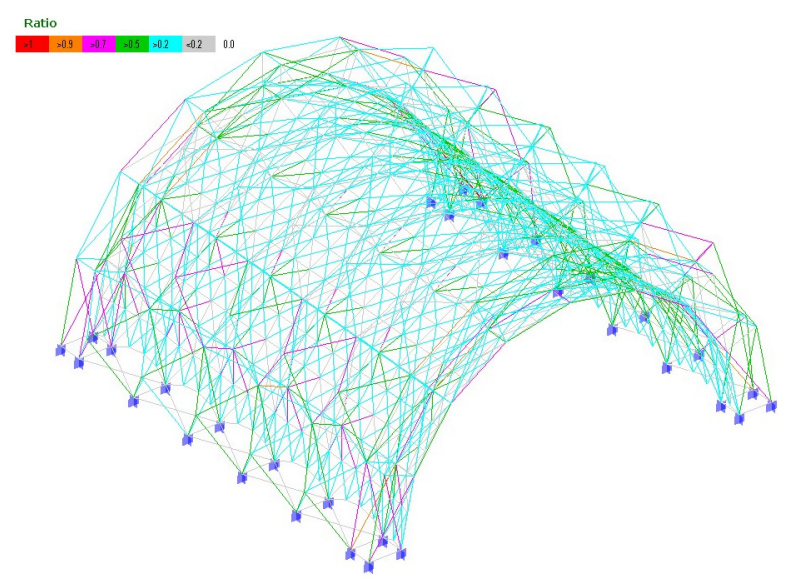

Figura 11. Ratios de seguridad de la Estructura Fuente: Extraído de la Revista de Arquitectura, Universidad Católica de Colombia, (Morales, 2016b, 2018a).

\section{DISCUSIÓN DE LOS RESULTADOS}

Una vez obtenido los resultados finales del anterior desarrollo de simulación estructural, se hace una discusión reflexiva sobre el tema, ya que hay muy pocos trabajos sobre el estudio de los sistemas transformables en la actualidad, para mencionar algunos investigadores. El Dr. Félix Escrig Pallares y el Dr. José Sánchez Sánchez de la Universidad de Sevilla, el Dr. Juan Gerardo Oliva Salinas de la Universidad Nacional Autónoma de México y el Dr. Carlos Hernández de la Universidad de Venezuela, son los que en algún momento desarrollaron referencias constructivas contemporáneas importantes de movilidad mecánica de sistema plegable en las cubiertas. Por esta razón, la presente investigación se justificó en la línea de tiempo y las tecnologías actuales.

Este estudio ayudo a crear un parteaguas sobre qué tipo de factores de carga podríamos ocupar en el diseño y análisis de las estructuras plegables. Esto generó una hipótesis de análisis estructural respaldadas por los métodos del Dr. Félix Escrig, que ayudaron a seguir una metodología de experimentación de análisis numérico de un sistema transformable, la cual se basó en la modelación y verificación por medio del software WinTess, tomando en cuenta las normas Load and Resistance Factor Design y Norma de la Construcción del Reglamento del Distrito Federal 
y materiales que pudieran mejorar el proceso de la construcción posteriormente; esto da como resultado un sistema estructural configurable a su entorno.

Todo esto deja diferentes líneas de investigación paralelas para abordar y generar más conocimiento teórico. Un ejemplo de ello es el uso material como el aluminio estructural, aluminio aeroespacial, plástico estructural y acero reforzado con fibra de vidrio, etc., por lo cual nos daría un bagaje de posibilidades de aplicaciones en la arquitectura, la aplicación de este mismo método, pero con otros sistemas plegables $u$ otros sistemas estructurales plegables combinados. La investigación nos brinda como resultado medular una propuesta metodología de experimentación en el cual se puede analizar modelos físicos más rápidos, que puedan ayudarnos a comprender como desarrollar los sistemas transformables. (Broto 2006) (Morales, 2016a, 2016b, 2017, 2018a, 2019) (Llorens, 2018)

\section{CONCLUSIÓN}

La investigación se basó desde el principio en desarrollar un análisis estructural de las cubiertas plegables en base a factores de cargas óptimos, encontrados en los manuales del LRFD (Load and Resistance Factor Design) y el NCRDF (Normas de Construcción del Reglamento del Distrito Federal) y el MDVCFE (Manual de Diseño por Vientos de la Comisión Federal de Electricidad), nuestra guía metodología de diseño fue sustentada por las hipótesis del Dr. Félix Escrig, ya que sus estudios encontrados sirvieron para realizar una simulación estructural de un sistema transformable.
Todo esto ofreció un parámetro para desarrollar una propuesta de cálculo experimental para el diseño de una cubierta plegable, los cuales sirven para verificar y validar posteriormente prototipos o modelos. Definiendo la utilidad del sistema estructural, en este caso, una cubierta de tipo industrial de cubiertas no transitables, se aplican los factores de cargas a la simulación que se realizó por un software llamado WinTess que realiza un análisis de segundo orden, ya que este tipo de estructuras tiende a ser deformables por sus conexiones flexibles, dado que requieren de mecanismos en las uniones articuladas para materializar su movilidad y puedan retornar a su estado inicial. Finalmente, con los parámetros de combinaciones necesarias reglamentadas por las anteriores normas, con esto se tiene un planteamiento para proponer y definir aproximaciones de resultados óptimos para el diseño constructivo de prototipos (Figura 12 a la 19). (Morales 2012, 2013a, 2017, 2018a, 2019)

La utilización de un software especializado ayudo en gran medida a realizar el diseño estructural de una cubierta, llegando a generar varios parámetros y premisas para realizaron una configuración constructiva. Así como también, el considerar la membrana estructural desde un principio en el diseño, para conocer las tensiones reales que iban a interactuar en la estructura plegable. Aunque no todo está concluido en esta investigación, hay puntos que tendrán que estudiarse más a fondo, y así realizar un estudio más detallado, como el de las conexiones constructivas de los nodos, los detalles constructivos de la membrana estructural con las conexiones de los nodos, el manejo de otros materiales más ligeros o el uso de materiales alternativos sustentable como el bambú, que nos dejara una gama diferente de configuraciones que pudiera tener una estructura plegable (Figura. 20 a la 27). (Morales 2013a, 2017, 2018a, 2019) 

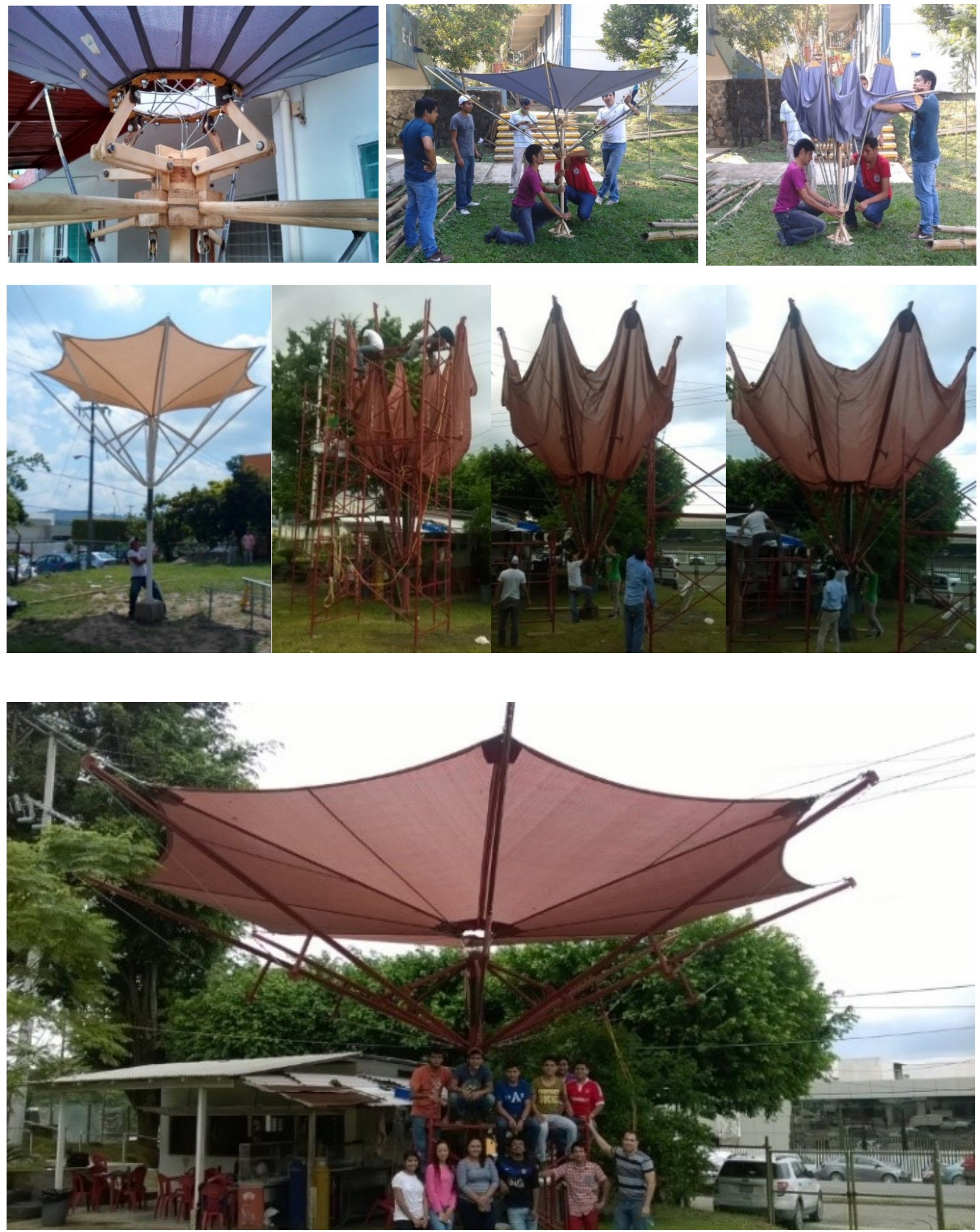

Figura 12 a la 19. Prototipados de sistemas plegables desarrollados en el Laboratorio de Estructuras Fuente: Extraídos de la Revista de Arquitectura, Universidad Católica de Colombia, (Morales 2016a, 2018a, 2017, 2019) 

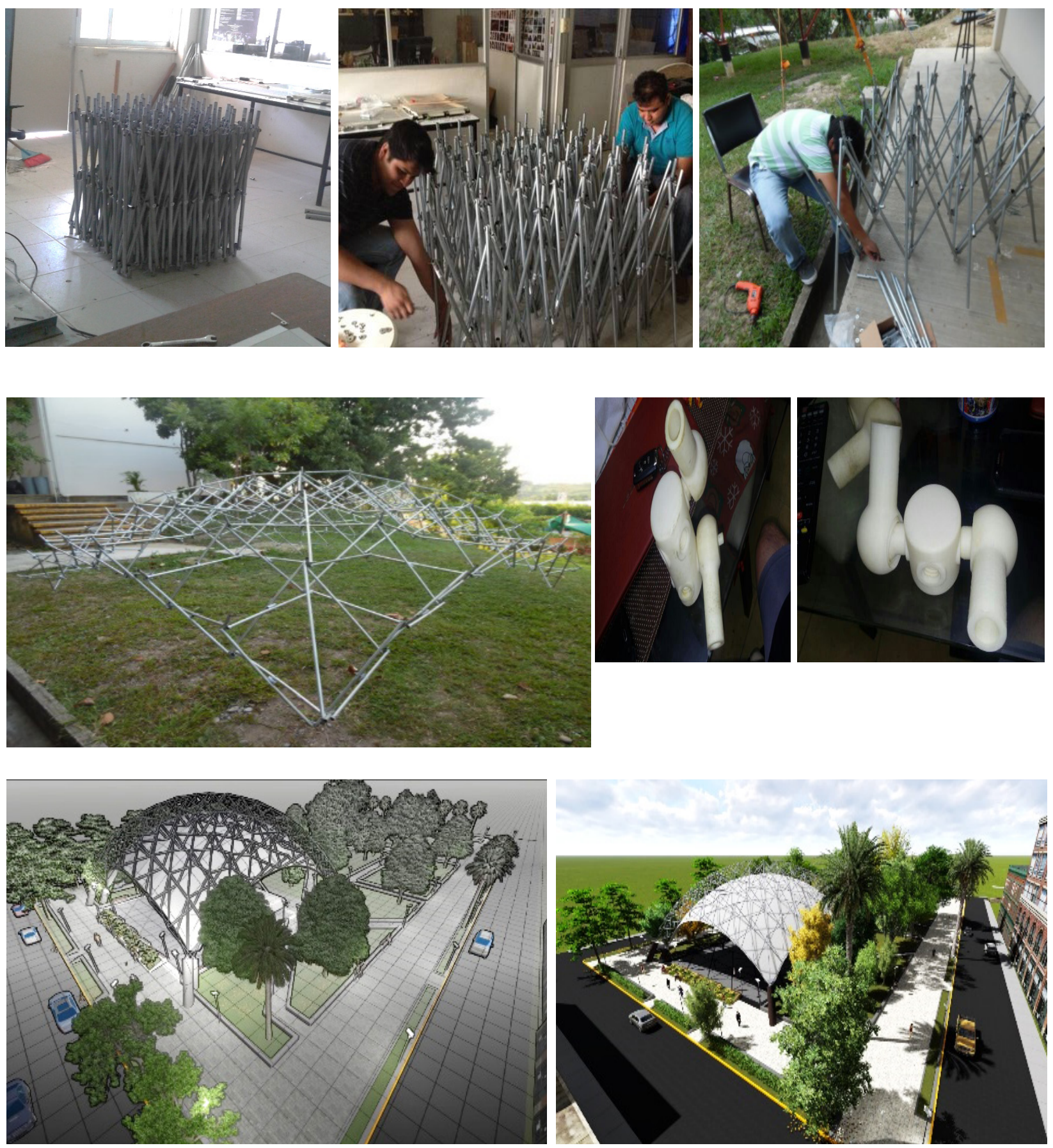

Figura. 20 a la 27. Propuestas para el Ayuntamiento para rescate de espacios públicos desarrollados por vinculación con el Laboratorio de Estructuras

Fuente: Extraído de la Estancia Post Doctoral, (Morales 2012) 


\section{REFERENCIAS}

Berger, h. (1996)." Light Structures, Structures of Light, the Art of Tensile Architecture." 1a Edition. Suiza: Basel.

Broto, c. (2006). Cubiertas, Innovación y Diseño. 1a, ed.: Editorial Links.

Candela, F., Piñero, e.p., Calatrava, s., Escrig, f. y Valcarcel, j.p. (1993), arquitectura

transformable, Publicación de la escuela técnica superior de Architectura de Sevilla, Sevilla.

Chilton, I.C., Choo, B.S. y Wilkinson, D. (1998), Un Análisis Paramétrico de la Geometría de Estructuras de Bastidor Reciprocas Retractiles. En los Procedimientos de la Conferencia Isa98 "Estructuras Livianas en Ingeniería de la Arquitectura y Construcción", vol.1, 547-555, Sydney, Australia.

Chilton, j. (2000)."Space Grid Structures", 1a ed, Editorial Architectural Press, Oxford, Boston, Usa.

Chudley, r. (2001). Manual de Construcción de Edificios. (3a ed.), Barcelona: Gustavo Gili, S.a. de C.V.

Escrig, F. (1988), Estructuras espaciales desplegables curvas, Revista Informes de la Construcción, Vol. 39, ISSN:1998-3234, Editorial Consejo Superior de Investigaciones Científicas.

Escrig, F. (2012). Modular, ligero, transformable: un paseo por la arquitectura ligera móvil. Ed, Editorial, Universidad de Sevilla. Sevilla: Universidad de Sevilla.

Grupo Estran. Estructuras Transformables Disponible en http://www.grupoestran.com. Consultado 20122014

IMCA (Instituto Mexicano de la Construcción de Acero) (2010), Manual de Construcción en Acero, "Diseño por Esfuerzo Permisibles". 6 edición, Editorial Limusa Noriega.

Llorens Duran, J.I. (2018). VIII Simposio Latinoamericano de Tenso-estructuras., Lima, Peru, 2018. Reglamentación Europea sobre Estructuras de Membranas.

Manual de diseño de WinTess3. Fuente: http://tecno.upc.edu/wintess/manual

Manual de diseño Estructural de CFE (Comisión Federal de Electricidad). http://cgservicios.df.gob.mx/ prontuario/vigente/385.htm.

McCormac, (2000). Diseño de Estructuras de Acero, Método LRFD, 2 edición, Editorial Alfaomega

Morales, C. C (2013a). Diseño de Cubiertas Plegables Tensadas en la Arquitectura. Tesis de Máster de Ingeniería para la Arquitectura, Área: Estructuras, Universidad Camilo José Cela, Madrid, España.

Morales, C. C (2013b) Prototipo de Diseño de una Cubierta Retráctil Tensada. Revista de Arquitectura, Vol. 15, ISSN: 2357-626X Editorial Universidad Católica de Colombia, Colombia.

Morales, C. C (2014) Proceso de diseño de sistemas transformables En las cubiertas ligeras. Revista Modulo Arquitectura, Vol. 14, ISSN: 2389-7732. Editorial Universidad de la Costa, Colombia.

Morales, C. C (2016a) Construcción Experimental de un sistema Transformable Tensado Plegable. Revista de Arquitectura, Vol. 18, ISSN: 2357-626X Editorial Universidad Católica de Colombia, Colombia. 
Morales, C. C (2016b) Development and experimental construction of a transformable roof (first phase). Magazine Asian Academic Research Journal of Multidisciplinary, Vol. 3, ISSN: 2319 - 2801. Editorial Asian Academic Research Associates de C.V.

Morales, C. C (2017) Desarrollo de la forma de una tenso estructura en el diseño por computadora. Revista Procesos Urbanos, Vol. 4, ISSN: 2500 - 5200. Editorial Corporación Universitaria del Caribe.

Morales, C. C (2018a) Diseño y desarrollo de patrones de la forma de una tenso-estructura. Revista de Arquitectura. Vol. 21, ISSN: 2357-626X Editorial Universidad Católica de Colombia, Colombia.

Morales, C. C (2018b) Diseño de una cubierta hiperbólica plegable tensada. Revista Procesos Urbanos, Vol. 5, ISSN: 2500 - 5200. Editorial Corporación Universitaria del Caribe.

Morales, C. C (2019a) Diseño y construcción de un paraguas plegable para espacios arquitectónicos. Revista de Arquitectura. Vol. 22, ISSN: 2357-626X Editorial Universidad Católica de Colombia, Colombia.

Morales, C. C, Mercado E. J (2019b) Proceso de diseño de una cubierta con el principio de tensegridad para espacios de esparcimiento. Revista Procesos Urbanos, Vol. 6, ISSN: 2500 - 5200. Editorial Corporación Universitaria del Caribe.

Morales, C. C. (2012). Diseño de Cubiertas Ligeras Tensadas. Estancia Post Doctoral, Universidad Politécnica de Catalunya.

Morales, C.C (2017). Experiencias y casos de estudio: construcción de una cubierta hiperbólica tensada para espacios tropicales. En Pesantez, G. y Flores, W. (comps.). Arquitectura: experiencias y propuestas para la ciudad (pp. 177-192). Guayaquil: Centro de Investigaciones y Desarrollo de Ecuador.

Normas Complementarias de Construcción de Distrito Federal. Fuente: https://www.isc.cdmx.gob.mx/ dependencia/marco-normativo Consultado 2009-2018

Segui W. (2000). Diseño de Estructuras de Acero con LRFD. 2a Ed. México: Editorial Thomson Editores, S.A 